2011-04

\title{
Designing clinical trials in older people
}

\author{
Carroll, Camille
}

http://hdl.handle.net/10026.1/3487

10.1016/j.maturitas.2011.02.002

Maturitas

Elsevier BV

All content in PEARL is protected by copyright law. Author manuscripts are made available in accordance with publisher policies. Please cite only the published version using the details provided on the item record or document. In the absence of an open licence (e.g. Creative Commons), permissions for further reuse of content should be sought from the publisher or author. 
Review

\title{
Designing clinical trials in older people
}

\author{
C.B. Carroll ${ }^{\mathrm{a}, *}$, J.P. Zajicek ${ }^{\mathrm{b}}$
}

a Peninsula College of Medicine and Dentistry, University of Plymouth, Dept. Clinical Neurobiology, John Bull Building, Tamar Science Park, Research Way, Plymouth, Devon, UK PL6 8BU

b Peninsula College of Medicine and Dentistry, University of Plymouth, Dept. Clinical Neuroscience, ITTC Building, Tamar Science Park, Plymouth, Devon, UK PL6 8BX

\section{A R T I C L E I N F O}

\section{Article history:}

Received 19 January 2011

Received in revised form 1 February 2011

Accepted 1 February 2011

\section{Keywords:}

Clinical trial design

Elderly

Recruitment

Retention

Trial analysis

\begin{abstract}
A B S T R A C T
Adequate medical care of the increasingly ageing population requires robust clinical trial data both to inform treatment decisions, and to understand the natural history of diseases which primarily affect the elderly. However, this information is widely lacking, which is likely to have significant clinical consequences. Under-representation of older people in clinical trials is well documented, the reasons including physicians' perception, protocol eligibility criteria, and functional status requirements. Many clinical trial designs remain conservative and there is no established standardised methodology for recruiting more elderly patients with co-morbidities and disability into clinical trials. Designing clinical trials in older people poses a unique set of challenges, particularly regarding recruitment, retention and data analysis. In this review we outline the difficulties encountered in conducting clinical trials in older patients and describe some of the initiatives that can be put in place to counteract them. It is only by addressing these challenges with careful and adequately resourced protocol design that clinical trials may successfully address the therapeutic questions raised by our ageing population.
\end{abstract}

(c) 2011 Elsevier Ireland Ltd. All rights reserved.

\section{Contents}

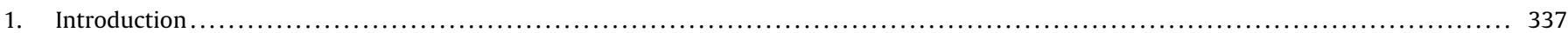

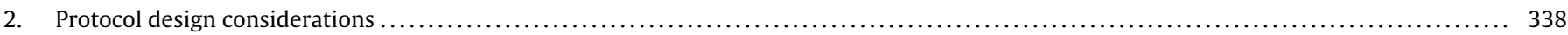

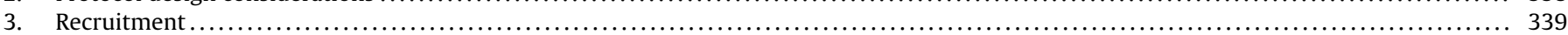

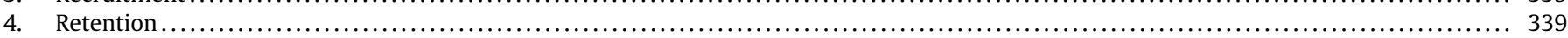

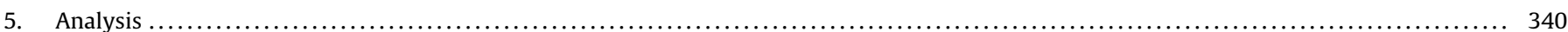

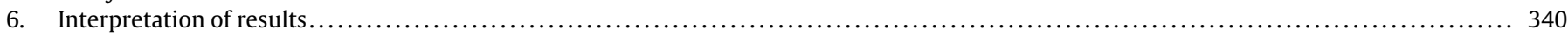

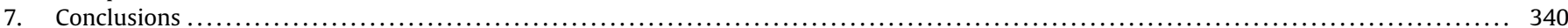

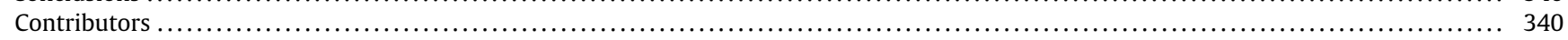

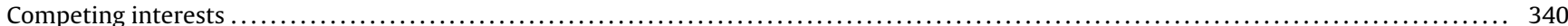

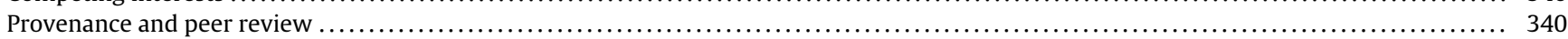

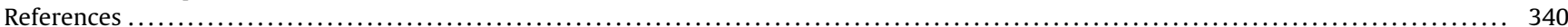

\section{Introduction}

The percentage of the UK population aged 65 years and over is projected to increase from $16 \%$ in 2009 to $23 \%$ in 2034 [1]. The largest increase is projected to be the very elderly (those aged 85 years and over), rising from 1.4 million in 2009 to 3.5 million in 2034 (5\% of the total population) [1]. This increase in age will be paralleled by increases in the prevalence of conditions affecting the

\footnotetext{
* Corresponding author. Tel.: +44 1752 437420; fax: +44 1752517846.

E-mail address: camille.carroll@pms.ac.uk (C.B. Carroll).
}

elderly such as dementia: the worldwide prevalence of Alzheimer's disease is forecast to quadruple by 2050 , affecting 1 in 85 people [2]. However, despite the need for trial data in the elderly population, it is widely lacking and it is likely that clinical treatment of the elderly is being limited, as the extrapolation of trial data from a younger population may not necessarily be valid [3]. Under-representation of older people in clinical trials is well documented [4,5], the reasons including physicians' perception, protocol eligibility criteria, and functional status requirements [6]. Inherent difficulties include frailty, such that participation would be too onerous, and cognitive decline leading to problems with consent and retention [21]. In addition various factors result in failure to meet inclusion criteria, 
Table 1

Factors that contribute to effective research in the older person.

\begin{tabular}{|c|c|}
\hline Successful strategies & Limiting factors \\
\hline \multicolumn{2}{|l|}{ General } \\
\hline Maximise patient involvement in all aspects of study design and implementation & Restrictive inclusion and exclusion criteria \\
\hline \multicolumn{2}{|l|}{ Minimise exclusion criteria } \\
\hline \multicolumn{2}{|l|}{$\begin{array}{l}\text { Anticipate nonresponse and attrition and consider measures to counteract, such as } \\
\text { oversampling or triggered sampling }\end{array}$} \\
\hline \multicolumn{2}{|l|}{ Careful selection of non-onerous validated outcome measures } \\
\hline \multicolumn{2}{|l|}{ Plan for change over time such as development of cognitive impairment } \\
\hline \multicolumn{2}{|l|}{ Have flexible dosing regimes in drug therapy trials, and plans to limit exclusion } \\
\hline \multicolumn{2}{|l|}{ Recruitment } \\
\hline Plan and resource several recruitment strategies & Clinician gate-keeping \\
\hline Consider pilot phase for recruitment strategy assessment & Time commitment required from recruiter \\
\hline Ongoing monitoring and adaptation of recruitment strategy & Potential burden of study \\
\hline Pay particular attention to needs of special groups e.g. ethnic minorities & Impaired literacy in the elderly \\
\hline Development of personal relationship between recruiter and participant & Audio-visual impairment \\
\hline Employ simple measures such as telephone follow-up of invitation letter & Participant mistrust of researchers \\
\hline Provision of information about studies to potential physician recruiters & Lack of research awareness amongst health care professionals \\
\hline Provision of research support and resource to physicians & Practitioner time in clinic \\
\hline \multicolumn{2}{|l|}{ Encourage endorsement of study by local health care practitioners } \\
\hline \multicolumn{2}{|l|}{ Retention } \\
\hline Develop strategy to cater for the specific needs of those at higher risk of attrition & Mobility limitations \\
\hline Ensure trial experience is positive & Poor health of participants \\
\hline Consider alternatives to study visits, such as home visits or telephone contact & Hospitalisations \\
\hline \multicolumn{2}{|l|}{ Develop flexible study schedule } \\
\hline \multicolumn{2}{|l|}{ Consider use of a proxy to provide follow-up data } \\
\hline \multicolumn{2}{|l|}{ Consistent researcher throughout study } \\
\hline \multicolumn{2}{|l|}{ Analysis } \\
\hline Consider pilot phase to estimate missing data rates and potential protocol modifications & Death or withdrawal \\
\hline Develop strategy to minimise missing data within study design & Incomplete completion of questionnaires or assessments \\
\hline \multicolumn{2}{|l|}{ Incorporate adequate statistical methodology for missing data } \\
\hline \multicolumn{2}{|l|}{ Adequately describe characteristics of sub-populations with missing data } \\
\hline \multicolumn{2}{|l|}{ Generalisability of results } \\
\hline Consider use of multidimensional assessment of function rather than chronological age & $\begin{array}{l}\text { Study population may be fitter with less co-morbity than the } \\
\text { general population }\end{array}$ \\
\hline
\end{tabular}

such as functional decline, polypharmacy, organ failure and institutionalisation. Other barriers include the extra time and resources needed to recruit older patients, and the extra support they may require to ensure retention. Even when older people are included in studies, selection bias occurs such that those who are healthier and with higher economic status tend to participate, casting doubt on the general applicability of the results [7].

These problems affect all branches of elderly medicine - from falls prevention to oncology. In some areas, such as oncology, there have been national initiatives to boost geriatric involvement in clinical trials and examine reasons why this is lacking, such as the Geriatric Oncology Consortium in the US. However, many clinical trial designs remain conservative and there is no established standardised methodology for recruiting more elderly patients with co-morbidities and disability into clinical trials.

Conducting a clinical trial involves several processes including protocol design, recruitment strategy, protocol implementation, retention, analysis and dissemination of results. In this review we outline the difficulties encountered in conducting clinical trials in older patients and describe some of the initiatives that can be put in place to counteract them (summarised in Table 1).

\section{Protocol design considerations}

In general, patient involvement should be sought for all stages of clinical trial design to inform protocol development and ensure its acceptability to the target population. To maximise inclusiveness, exclusion criteria should be kept to a minimum. Investigators should consider whether measures need to be put in place to counteract the difficulties associated with nonresponse or nonparticipation of patients who fulfil eligibility criteria, such as adopting alternative randomisation methods (e.g. cluster randomisation) [7]. When selecting outcome measures, it is important to ensure that as far as achievable, scales and assessment tools have been validated for use in the target population, for example self-completion scales developed in a younger population may have a form of wording with which a more elderly participant may not identify [8]. It is also important to ensure that the outcome measure selected is appropriate for the stage of the disease it is being used to evaluate. For example, the Alzheimer's disease assessment scale - cognitive subscale (ADAS-cog) was developed for the assessment of Alzheimer's dementia, and is now increasingly used in earlier stages of the illness as early clinical diagnosis and interventions are being evaluated. However, it has been demonstrated the sensitivity of the scale is significantly compromised at earlier disease stages, with pronounced ceiling effects in several components [9], thereby limiting its appropriateness for use in these sorts of studies.

Longitudinal studies have their own considerations [10] such as the development of cognitive impairment during the course of the study for which a plan must be in place in order to secure ongoing informed consent. In addition there may be changes in the study protocol, equipment or software used during the course of the study that need to be taken into account. Missing data are inevitable in longitudinal studies involving the elderly and there needs to be a clear plan in place to manage this, either by appropriate statistical methods or by planned missing designs [11]. In addition the extent of missing data can be minimised by reducing time required to complete the dataset by incorporating adaptive data collection techniques such as item response theory (IRT) where banks of items are ordered hierarchically in terms of difficulty, or computer-adapted testing (CAT) where the testing session is adapted to each participant [12]. These techniques also have the advantage of reducing floor and ceiling effects of the outcome measures selected.

Trials of drug therapies are potentially more difficult to perform in the elderly because of problems with altered pharmacokinet- 
ics, particularly with regard to reduced volume of distribution and reduced renal excretion [33], which may result in increased drug toxicity and necessitate dose adjustment. Other potential confounders in the elderly are anaemia, altered hepatic metabolism, and drug-drug interactions from pre-existing medication regimes. Pharmacodynamic considerations may also be relevant in the elderly such as altered catabolism, enzymatic clearance or cellular transport mechanisms. Nevertheless in order to determine the benefit/risk balance for any individual patient, it is important that strategies are put in place to allow for recruitment of these patients to clinical trials, such as GFR-dependent dose adjustment or treatment of anaemia with erythropoietin. Failure to maximise inclusiveness in recruitment will result in failure to recognise all those who may benefit most from the proposed therapy.

\section{Recruitment}

Difficulties with recruitment can generally be divided into those which affect clinical trials in general, such as issues with clinician gate-keeping, potential burden and time-commitment and those which are specific to a more elderly population, such as impaired literacy and audio-visual impairment. Several studies have investigated recruitment strategies in the elderly and some common principles emerge [13-18], particularly the need to plan and resource several recruitment initiatives, have an adaptive flexible recruitment strategy, be aware of individual needs or those of particular groups such as ethnic minorities [19], and develop a personal relationship between researcher and patient to maximise trust. The establishment of trust between participant and researcher is particularly important in qualitative research where older patients may be reluctant to discuss their experiences with younger researchers or may feel a greater sense of stigma associated with the condition being investigated [8].

Few studies have been designed specifically to investigate and compare recruitment strategies for older adults. However, a systematic review [20] has highlighted simple measures that can be implemented such as follow-up of an invitation letter with a telephone call, including trial documentation with the post-card reminder and including a pencil with questionnaires for completion.

Recruitment strategies should be targeted specifically and can range from advertisements in the media to face-to-face recruitment by the patient's own physician. In general face-to-face strategies are more effective and can help overcome patient concerns and gain acceptance and trust [15]. This is of particular relevance when recruiting to registers where it has been shown that age is a predictor of recruitment to a research registry [21], with a significantly lower level of recruitment in those aged over 60, reducing further with each decade. In addition, conducting community-based or epidemiological research may also require face-to-face recruitment as telephone recruitment or posted material is less likely to be effective in a population with a higher incidence of visual and auditory impairment. Recruitment to this type of study can also be boosted by forging links with charitable organisations representing the elderly, or relevant community leaders or organisations.

Although physician-based face-to-face recruitment can be a successful strategy, it does depend on the physician being aware of potential trials, being willing to recruit patients to studies and having the time to do so. Within the context of a busy service, these may remain aspirations. However, within the UK there are strategies in place to help overcome some encountered difficulties. Inclusion of patients within the NHS in research and the importance of the practice of research as a core NHS activity are receiving increasing recognition [22]. Infrastructure now exists to assist with deliver- ing research opportunities to all patients within the NHS, delivered via the research networks. Nevertheless, the process still requires clinicians being aware of and willing to participate in the research process. In specialties with well-established academic links, such as neurology, or those who have a strong culture of research, such as oncology, this poses less of a problem, but in other specialities the lack of research-interested or research-aware clinicians is a limitation. The decreasing number of academic departments of geriatric medicine may be a factor in reduced research awareness in the UK [23] There is also the potential that the physician may act as a 'gate-keeper' [24], thereby disenfranchising older patients, despite evidence that patients themselves are willing to participate in clinical studies [6]. In one survey of physicians the main reasons endorsed for not recruiting potentially eligible older patients to trials were found to be transportation needs, comorbid conditions not excluded by the eligibility criteria, but which the physician believed would adversely affect the patient, toxicity of the treatment, and patient ability to understand the trial [25]. Although changing physicians' perceptions to inclusion of older patients in trials may not be straightforward [26], it is important that this is not neglected, as endorsement of a study by the patient's own physician can be a powerful recruitment tool [27].

Ongoing monitoring of the success of the initial recruitment method and adaptability to deal with encountered difficulties should form part of the study design. This may well lead to an increased resource requirement for the recruitment phase of the study [14], with one published estimate of recruitment cost being $\$ 255.40$ per completing participant [19]. It may therefore be worthwhile considering a pilot phase to investigate alternative recruitment strategies.

\section{Retention}

Dropout during a trial can adversely affect the outcome of the study: the reduction in numbers may reduce the power, or the remaining sample may introduce bias. When designing a study measures should therefore be put in place both to maximise retention and to prevent bias.

It has been demonstrated that participants who are younger, perform better cognitively and are more highly educated have higher retention rates [21]. It is therefore important to identify those individuals who have a higher risk of attrition, which includes the elderly, and ensure strategies are in place to cater for their specific needs. A simple principle is to ensure that the trial experience is positive and beneficial, safe, in a readily accessible physical environment. The benefit of the trial may not necessarily be the possibility of being allocated a potentially beneficial therapy; it might be personal education or a sense of helping others [28]. With regard to retention, a qualitative study by Schlenk [28] identified 5 factors which contribute positively: the belief of an obligation to complete what has been started; reciprocity to the medical community; test results being of value in ongoing medical management; a health advantage even if by increased awareness or education; and socialization. This last is not to be underestimated in a population in which social isolation is common. A particular factor which can adversely affect retention of older trial participants is mobility limitation which can affect $26 \%$ of those aged between 65 and 74 years of age [28]. Measures can be put in place during the design phase of the protocol that will assist with retention of this group: avoidance of prolonged and numerous site visits, study visits carried out in locations with convenient parking and access, provision of transportation where appropriate, telephone or home visits where possible.

Factors that increase likelihood of attrition are taking part in more than one study, increasing age, being in poorer health, cogni- 
tive impairment and length of follow-up [29]. Means to overcome this may include oversampling of these groups in the initial phase to ensure sufficient numbers at follow-up, or to utilise triggered sampling designs. In addition, protocols should have the facility for flexibility regarding study dates, so that they can be rearranged if the participant is unwell; facilitate the presence of a family member to assist with interviews and study visits; obtain permission to contact a proxy to help locate the patient at the time of follow-up, or to contribute follow-up data; use the same researcher throughout the study if possible [30].

\section{Analysis}

Analysis of studies involving elderly participants poses a unique set of challenges due to the nature of the study population and the types of study being performed. Indeed it has been proposed that a subdiscipline of 'gerontologic biostatistics' be developed to facilitate development of analytical techniques and training of future researchers [31]. Challenges which require statistical attention include the use of multiple interventions and outcomes, health state transitions, floor and ceiling effects of outcome measures and missing data.

The problem of missing data affects all studies but is likely to be greater in trials which involve the elderly, due to factors such as incomplete completion of questionnaires, physical and cognitive problems limiting ability to complete assessments, and loss to follow-up limiting the internal and external validity of the results [32]. Strategies to minimise missing data should be developed and incorporated into all phases of study design and have been comprehensively reviewed by Hardy et al. [32]. In general strategies include defining readily obtainable primary outcome measures, prioritizing data collection, developing alternative data collection strategies and ensuring adequate allocation of resources. Designing a study to maximise data collection will involve balancing the competing requirements of minimizing exclusions and maximising retention and data completion. In addition it is important to accurately collect and describe the reasons for missing data or protocol deviations, and the characteristics of these study sub-populations. This will assist with interpreting the generalisability of the study findings. As with development of recruitment strategies, incorporating a pilot phase can assist with providing estimates of missing data rates and identification of study procedures or outcomes which may require modification, as well as determining the reliability of any proxy-completed measures. Missing data can be defined as missing completely at random (MCAR), missing at random (MAR) or missing not at random (MNAR). Although most missing data fall into the last category, most standard statistical and analytical compensatory techniques assume the data are MCAR or MAR. Appropriate statistical methods should therefore be incorporated into the analysis plan to deal with missing data whilst minimising introduction of bias [11].

\section{Interpretation of results}

Solutions to the problem of making trial data derived from younger, fitter elderly participants more generalisable to the less well population include the use of geriatric assessment questionnaires that describe the degree of co-morbidity and general health in the study population. Geriatric assessments of domains such as levels of cognitive dysfunction, and number and severity of comorbidities are known to be associated with patients' tolerance of new treatments and long-term survival and as such are performed explicitly or implicitly when making therapeutic decisions. However, such assessments rarely form part of clinical trial protocols.
In order to determine whether special measures are likely to be required in recruitment, retention or trial protocol with particular patients it is useful to have a multidimensional measure rather than chronological age. One such measure is the comprehensive geriatric assessment (CGA) which assesses function, comorbidity, socioeconomic conditions, cognition, emotional conditions, pharmacy, nutrition and geriatric syndromes [33]. Using the CGA has been reported to allow identification of 3 groups of patients: those who are functionally independent without serious comorbidity; those requiring assistance with one or more ADLs or with one or two comorbid conditions; and those who are frail. Adoption of an assessment tool such as the CGA for all patients over the age of 70 years would serve the dual purposes of: (1) the identification of new problems specific to the individual which may limit their trial participation; and (2) allowing assessment to be made of the generalisability of trial results to other patient groups. Another proposed measure is the geriatric minimum data set (GMDS-25) [5], the purpose of which is to provide standardisation of nomenclature, assessment tools and diagnostic criteria. Standardisation of reporting would have the additional advantage of facilitating comparative studies and meta-analyses.

Reporting of the characteristics of responding and nonresponding nonparticipants will also assist in assessing the generalisability and interpretation of the study results, as those that do not respond are more likely to have significant co-morbidities or be hospitalised, and may be the group most likely to benefit from the investigated intervention. If there is significant non-response it may be that the results cannot be generalised even to others that meet eligibility criteria [7].

\section{Conclusions}

Designing clinical trials in older people poses a unique set of challenges, particularly regarding recruitment, retention and data analysis. It is only by addressing these challenges with careful and adequately resourced protocol design that clinical trials may successfully address the therapeutic questions raised by our ageing population.

\section{Contributors}

Dr Camille Carroll and Prof. John Zajicek - writing and final editing of manuscript.

\section{Competing interests}

None declared.

\section{Provenance and peer review}

Commissioned and externally peer reviewed.

\section{References}

[1] ONS. National statistics online - ageing. http://www.statistics.gov.uk/cci/ nugget.asp?ID=949 2011.

[2] Brookmeyer R, Johnson E, Ziegler-Graham K, Arrighi HM. Forecasting the global burden of alzheimer's disease. Alzheimer's and Dementia 2007;3(3):186-91.

[3] Petersen LK, Christensen K, Kragstrup J. Lipid-lowering treatment to the end? A review of observational studies and rcts on cholesterol and mortality in 80+year olds. Age and Ageing 2010;39(6):674-80.

[4] Hutchins LF, Unger JM, Crowley JJ, Coltman CA, Albain KS. Underrepresentation of patients 65 years of age or older in cancer-treatment trials. New England Journal of Medicine 1999;341(27):2061-7.

[5] Van Kan GA, Sinclair A, Andrieu S, Rikkert MO, Gambassi G, Vellas B. The geriatric minimum data set for clinical trials (gmds). Journal of Nutrition Health \& Aging 2008;12(3):197-200.

[6] Townsley CA, Selby R, Siu LL. Systematic review of barriers to the recruitment of older patients with cancer onto clinical trials. Journal of Clinical Oncology 2005;23(13):3112-24. 
[7] Vind AB, Andersen HE, Pedersen KD, Jorgensen T, Schwarz P. Baseline and follow-up characteristics of participants and nonparticipants in a randomized clinical trial of multifactorial fall prevention in denmark. Journal of the American Geriatrics Society 2009;57(10):1844-9.

[8] Shellman J, Mokel M. Overcoming barriers to conducting an intervention study of depression in an older African American population. Journal of Transcultural Nursing 2010;21(4):361-9.

[9] Cano SJ, Posner HB, Moline ML, et al. The adas-cog in alzheimer's disease clinical trials: psychometric evaluation of the sum and its parts. Journal of Neurology Neurosurgery \& Psychiatry 2010;81(12):1363-8.

[10] Newman AB. An overview of the design, implementation, and analyses of longitudinal studies on aging. Journal of the American Geriatrics Society 2010;58:S287-91.

[11] Palmer RF, Royall DR. Missing data? Plan on it! Journal of the American Geriatrics Society 2010;58:S343-8.

[12] Gill TM. Assessment of function and disability in longitudinal studies. Journal of the American Geriatrics Society 2010;58:S308-12.

[13] Bonk J. A road map for the recruitment and retention of older adult participants for longitudinal studies. Journal of the American Geriatrics Society 2010;58:S303-7.

[14] Adams J, Silverman M, Musa D, Peele P. Recruiting older adults for clinical trials. Controlled Clinical Trials 1997;18(1):14-26.

[15] Samelson EJ, Kelsey JL, Kiel DP, et al. Issues in conducting epidemiologic research among elders: lessons from the mobilize boston study. American Journal of Epidemiology 2008;168(12):1444-51.

[16] Forster SE, Jones L, Saxton JM, et al. Recruiting older people to a randomised controlled dietary intervention trial - how hard can it be? BMC Medical Research Methodology 2010:10.

[17] Sanders KM, Stuart AL, Merriman EN, et al. Trials and tribulations of recruiting 2000 older women onto a clinical trial investigating falls and fractures: vital d study. BMC Medical Research Methodology 2009:9.

[18] Mody L, Miller DK, McGloin JM, et al. Recruitment and retention of older adults in aging research. Journal of the American Geriatrics Society 2008;56(12):2340-8.

[19] Lindenstruth KA, Curtis CB, Allen JK. Recruitment of African American and white postmenopausal women into clinical trials: the beneficial effects of soy trial experience. Ethnicity \& Disease 2006;16(4):938-42.

[20] Auster J, Janda M. Recruiting older adults to health research studies: a systematic review. Australasian Journal on Ageing 2009;28(3):149-51.
[21] Newberry A, Sherwood P, Hricik A, et al. Understanding recruitment and retention in neurological research. Journal of Neuroscience Nursing 2010;42(1):47-57.

[22] NIHR. Being a good research partner: the virtues and rewards. NHS Confederation Briefing 2010; October 2010, 207(207).

[23] Bartram L, Crome P, McGrath A, Corrado OJ, Allen SC, Crome I. Survey of training in geriatric medicine in UK undergraduate medical schools. Age and Ageing 2006;35(5):533-5.

[24] Sharkey K, Savulescu J, Aranda S, Schofield P. Clinician gate-keeping in clinical research is not ethically defensible: an analysis. Journal of Medical Ethics 2010;36(6):363-6.

[25] Kornblith AB, Kemeny M, Peterson BL, et al. Survey of oncologists' perceptions of barriers to accrual of older patients with breast carcinoma to clinical trials. Cancer 2002;95(5):989-96.

[26] Cohen HJ. Overturning barriers will take heavy lifting. Journal of Oncology Practice 2008;4(4):169-70.

[27] Basche M, Barón AE, Eckhardt SG, et al. Barriers to enrollment of elderly adults in early-phase cancer clinical trials. Journal of Oncology Practise 2008;4(4): $162-8$.

[28] Schlenk EA, Ross D, Stilley CS, Dunbar-Jacob J, Olshansky E. Research participation among older adults with mobility limitation. Clinical Nursing Research 2009;18(4):348-69.

[29] Chatfield MD, Brayne CE, Matthews FE. A systematic literature review of attrition between waves in longitudinal studies in the elderly shows a consistent pattern of dropout between differing studies. Journal of Clinical Epidemiology 2005;58(1):13-9.

[30] Puts MTE, Monette J, Girre V, et al. Participation of older newly-diagnosed cancer patients in an observational prospective pilot study: an example of recruitment and retention. BMC Cancer 2009:9.

[31] Van Ness PH, Charpentier PA, Ip EH, et al. Gerontologic biostatistics: the statistical challenges of clinical research with older study participants. Journal of the American Geriatrics Society 2010;58(7):1386-92.

[32] Hardy SE, Allore H, Studenski SA. Missing data: a special challenge in aging research. Journal of the American Geriatrics Society 2009;57(4): 722-9.

[33] Balducci L, Extermann M. Management of cancer in the older person: a practical approach. Oncologist 2000;5(3):224-37. 